\title{
RESEARCH
}

Open Access

\section{Discordance between the triglyceride glucose index and HOMA-IR in incident albuminuria: a cohort study from China}

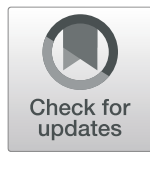

Wei Gao ${ }^{1 \dagger}$, Jialu Wang ${ }^{2,3+}$, Yan Chen ${ }^{1 \dagger}$, Hongmei Qiao ${ }^{1}$, Xiaozhong Qian ${ }^{1}$, Zhuojun Xin ${ }^{2,3}$, Zhiyun Zhao ${ }^{2,3}$ Tiange Wang ${ }^{2,3}, \mathrm{Yu} \mathrm{Xu}^{2,3}$, Min Xu $\mathrm{u}^{2,3}$, Yufang $\mathrm{Bi}^{2,3}$, Mian $\mathrm{Li}^{2,3^{*}}$ and Jinli Gao ${ }^{1 *}$

\begin{abstract}
Background: To date, there have no study comparing the associations between TyG index and HOMA-IR on the risk of incident albuminuria. Accordingly, the objective of the present study is to use discordance analysis to evaluate the diverse associations between TyG index and HOMA-IR on the risk of incident albuminuria.

Methods: A community-based prospective cohort study was performed with 2446 Chinese adults. We categorized participants into 4 concordance or discordance groups. Discordance was defined as a TyG index equal to or greater than the upper quartile and HOMA-IR less than the upper quartile, or vice versa.

Results: During a median follow-up period of 3.9 years, 203 of 2446 participants developed incident albuminuria (8.3\%). In the multivariable logistic analyses, the high TyG index tertile group was associated with a 1.71-fold (95\% confidence interval (Cl) 1.07-2.72) higher risk of incident albuminuria, comparing with the low tertile group.

Participants in TyG (+) \& HOMA-IR (-) group had a greater risk of incident albuminuria compared with those in TyG $(-)$ \& HOMA-IR (-) group after multivariate adjustment. Subgroup analyses showed that low HOMA-IR and discordantly high TyG index was closely related to a highest risk of incident albuminuria in cardiovascular metabolic disorder subjects.

Conclusions: Participants with a discordantly high TyG index had a significantly greater risk of incident albuminuria, especially in metabolic dysfunction subjects. The TyG index might be a better predictor of early stage of chronic kidney disease than HOMA-IR for subjects with metabolic abnormality.
\end{abstract}

Keywords: Chronic kidney disease, Discordance analysis, Insulin resistance, Triglyceride-glucose index

\footnotetext{
* Correspondence: limian39@aliyun.com; 390795950@qq.com

tWei Gao, Jialu Wang and Yan Chen contributed equally to this work. ${ }^{2}$ Department of Endocrine and Metabolic Diseases, Shanghai Institute of Endocrine and Metabolic Diseases, Ruijin Hospital, Shanghai Jiaotong University School of Medicine, 197 Rui-Jin 2nd Road, Shanghai 200025, China

${ }^{1}$ Songnan Town Community health Service Center, Baoshan District, Shanghai, China

Full list of author information is available at the end of the article
}

(c) The Author(s). 2021 Open Access This article is licensed under a Creative Commons Attribution 4.0 International License, which permits use, sharing, adaptation, distribution and reproduction in any medium or format, as long as you give appropriate credit to the original author(s) and the source, provide a link to the Creative Commons licence, and indicate if changes were made. The images or other third party material in this article are included in the article's Creative Commons licence, unless indicated otherwise in a credit line to the material. If material is not included in the article's Creative Commons licence and your intended use is not permitted by statutory regulation or exceeds the permitted use, you will need to obtain permission directly from the copyright holder. To view a copy of this licence, visit http://creativecommons.org/licenses/by/4.0/ The Creative Commons Public Domain Dedication waiver (http://creativecommons.org/publicdomain/zero/1.0/) applies to the data made available in this article, unless otherwise stated in a credit line to the data. 


\section{Background}

Chronic kidney disease (CKD), a major disease burden, affects 8 to $16 \%$ of the population worldwide [1, 2]. Globally, metabolic disorders are the most common reasons for CKD [3]. Since extensive evidence has confirmed the strong association between CKD and an increased risk of cardiovascular disease $[4,5]$. Dysregulated metabolic factors, including diabetes mellitus, hypertension and dyslipidaemia, play leading roles in mediating this relationship, the early identification of CKD is critical for preventing clinical cardiovascular events. Additionally, large-scale studies have proven that albuminuria is a sensitive biological marker of progression of kidney diseases in early stage of CKD [6], and increased urinary albumin excretion is also an important indicator of cardiovascular metabolic risk factors [7, 8].

Insulin resistance (IR) is an early metabolic change in individuals with CKD [9]. Since the hyperinsulinaemiceuglycaemic clamp (HIEC) is a well-accepted 'gold standard' approach for evaluating IR, the homeostasis model assessment of IR (HOMA-IR) is a relatively most widely used tool to assess IR. Moreover, considering the convenience of implementation, researchers often use the upper quartile of HOMA-IR as the standard in population research. With regard to triglycerides (TGs) and high-density lipoprotein (HDL) cholesterol are components of metabolic disorders [10]. Previous studies have reported that lipid ratios, such as TG/HDL cholesterol, the non-HDL cholesterol/HDL cholesterol and triglyceride-glucose (TyG) index, are good indicators of the early identification of IR and have been widely used in clinical practice [11]. Moreover, the TyG index, calculated by fasting glucose and triglycerides, has been shown to perform better than HOMA-IR [12] and to be significantly correlated with HIEC [13].

To date, this kind of studies have rare explored the connection of the TyG index and albuminuria. A community-based study designed by Zhao et al. [14] recently showed that, in elderly individuals, higher levels of the TyG index were closely related to a greater risk of CKD and microalbuminuria. However, no studies have compared the abilities of the TyG index and HOMA-IR to measure new-onset albuminuria in the general population. Therefore, the present study was carried out to use discordance analysis for the assessment and further comparison of the effects of TyG index and HOMA-IR on incident albuminuria risk.

\section{Methods}

\section{Study design and participants}

A community-based investigation was undertaken from June to August 2009 in the Songnan community, Baoshan District, Shanghai, China. A circumstantial introduction of this research population has been previously published $[15,16]$. In total, 4012 participants underwent this examination at baseline. Serum and urine specimens were collected to detect TyG and urinary albumin-to-creatinine ratio (UACR). All participants were asked for to take part in a follow-up visit, of which 2883 individuals attended and tested for UACR between March and May in 2013. This current research was designed to explore the relationship of the TyG index and HOMA-IR to new-onset albuminuria. The exclusion criteria for the current analysis were subjects who (a) had self-reported kidney diseases at baseline $(n=36)$; (b) had $\mathrm{UACR} \geq 30 \mathrm{mg} / \mathrm{g}$ or estimated glomerular filtration rate $($ eGFR $)<60 \mathrm{~mL} / \mathrm{min}$ per $1.73 \mathrm{~m}^{2} \quad(n=390)$; (c) lacked UACR $(n=5)$; and (d) had missing data for TyG index or HOMA-IR $(n=6)$. Finally, 2446 individuals were included in this current study (Fig. 1).

The upper quartiles of baseline for the TyG index and HOMA-IR were calculated to classify participants into following 2 classes: low (lower than the upper quartiles) and high (equal to or higher than the upper quartiles). Then, participants were divided into 4 groups on the basis of the low/high value of the TyG index and HOMA-IR, as follows: TyG (-) \& HOMA-IR (-), TyG $(+)$ \& HOMA-IR (-), TyG (-) \& HOMA-IR (+) and TyG $(+)$ \& HOMA-IR $(+)$ groups. Discordance groups were presented as the TyG (+) \& HOMA-IR $(-)$ and TyG (-) \& HOMA-IR (+) groups.

The study protocol was approved by the Institutional Review Board of Rui Jin Hospital, Shanghai Jiao Tong University School of Medicine. All participants have written informed consent.

\section{Data collection and measurements}

The trained physicians used a normalized questionnaire to collect information including sociodemographic features, education levels, lifestyle and history of chronic disease with two face-to-face interviews. The status of current smoking or drinking was defined as smoking or drinking frequently in the past half year. The International Physical Activity Questionnaire was often used to evaluate the degrees of physical activity [17]. Body weight, height and blood pressure (BP) were measured by experienced nurses on the basis of a standard protocol [15]. Participants were asked to rest for $5 \mathrm{~min}$, and their seated blood pressure was measured three times on a nondominant arm with a $1 \mathrm{~min}$ interval. The average value of blood pressure was applied in the following analysis. Pulse pressure (PP) was obtained as the mean of three measurements by subtracting diastolic BP (DBP) from systolic BP (SBP).

Since the detection methods and instruments of blood samples and first-voided urine samples at early morning were previously described in published studies, repeat specification was no longer required here $[15,18,19]$. 


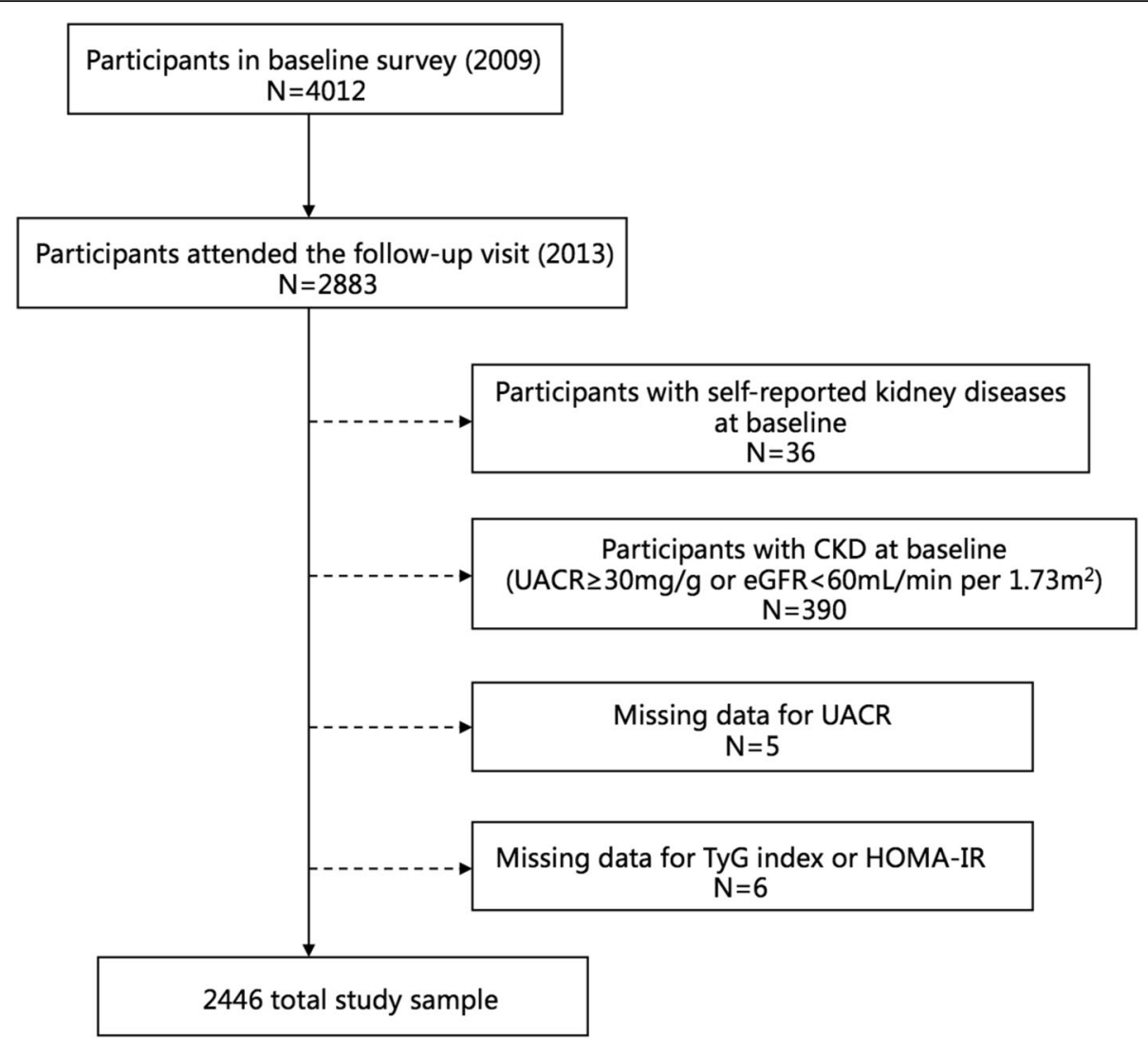

Fig. 1 Participant Flow Diagram of the Study. TyG, triglyceride glucose; HOMA-IR, homeostasis model assessment for insulin resistance; CKD, chronic kidney disease; UACR, urinary albumin-to-creatinine ratio; eGFR: estimated glomerular filtration rate

The TyG index was obtained by the formula: $\ln$ (fasting triglycerides $(\mathrm{mg} / \mathrm{dL}) \times$ fasting glucose $(\mathrm{mg} / \mathrm{dL}) / 2)$ [13] The HOMA-IR index was obtained as fasting insulin $(\mathrm{IU} / \mathrm{mL}) \times$ fasting glucose $(\mathrm{mmol} / \mathrm{L}) / 22.5[20]$.

\section{Definitions}

New-onset albuminuria was regarded as a UACR level of $30 \mathrm{mg} / \mathrm{g}$ or higher. The definition of CKD was an eGFR $\leq 60 \mathrm{~mL} / \mathrm{min}$ per $1.73 \mathrm{~m}^{2}$ or albuminuria. Hypertension was accepted as a SBP level of $140 \mathrm{mmHg}$ or higher, DBP level of $90 \mathrm{mmHg}$ or higher, or self-reported previous history of hypertension by professionals. The definition of diabetes was a fasting plasma glucose (FPG) level of $7.0 \mathrm{mmol} / \mathrm{L}$ or higher, 2-h glucose after $75-\mathrm{g}$ oral glucose tolerance test (OGTT) level of $11.1 \mathrm{mmol} / \mathrm{L}$ or higher, glycated haemoglobin (HbA1c) level of $6.5 \%$ or higher, or self-reported diagnosis by physicians and taking hypoglycaemic medications on the basis of the 2010 American Diabetes Association (ADA) criteria.

\section{Statistical analysis}

All data were analysed on the SAS version 9.4 platform (SAS Institute, Cary, NC, USA). A two-tailed $P$ value < 0.05 was considered statistically significant. Baseline variables were compared according to 4 concordance or discordance groups. Continuous data were shown as means \pm standard deviation, while categorical variables were displayed as numbers (\%). Differences in baseline characteristics among the 4 concordance or discordance groups were carried out by one-way analysis of variance or the $x^{2}$ test.

The relationships of the TyG index tertiles, HOMA-IR tertiles and the 4 concordance or discordance groups with new-onset albuminuria were explored using multivariate-adjusted logistic regression models. Covariates involved in the analysis included age, sex, status of current smoking or drinking, education levels, physical activity, HbA1c, PP, HDL cholesterol, LDL cholesterol, total cholesterol, BMI and medication usage of angiotensin-converting enzyme inhibitors (ACEIs) or angiotensin receptor blockers (ARBs).

Furthermore, stratified analysis of the 4 concordance or discordance groups with the risk of newonset albuminuria was repeated according to the status of diabetes, hypertension and age categories ( $\geq 60$ years old or $<60$ years old).

Additionally, the above analyses were repeated on the outcome of incident CKD. The flow diagram was shown 
in Supplementary Fig. 1, and the results were also shown in the supplementary materials.

\section{Results}

Baseline characteristics of participants in 4 concordance or discordance groups according to the TyG index and HOMA-IR

Baseline demographic and clinical characteristics were compared across the 4 concordance or discordance groups according to low or high categories for the TyG index and HOMA-IR (Table 1). The average age of enrolled subjects was 59.17 years old, and 968 of them were men (39.6\%). Age, current smoking status, education level, diabetes, hypertension, and dyslipidaemia were different among the 4 groups. Furthermore, participants in TyG (-) \& HOMA-IR (+) group were more probable to get a higher body mass index (BMI), SBP, DBP, FPG, fasting insulin, post load glucose and LDL cholesterol in comparison with TyG (+) \& HOMA-IR (-) group and had a higher prevalence of diabetes and hypertension. Scatterplots and prevalence of discordance and concordance defined according to the upper quartile values of TyG index and HOMA-IR were depicted in Supplementary Fig. 2.

Relationship of the TyG index, HOMA-IR and concordance or discordance groups with new-onset albuminuria and

\section{CKD}

Table 2 shows the odds ratios (ORs) of newonset albuminuria in participants according to TyG index

Table 1 Baseline characteristics of the concordance and discordance according to low or high TyG index and HOMA-IR categories

\begin{tabular}{|c|c|c|c|c|c|c|}
\hline & Total & TyG(-)\&HOMA-IR(-) & TyG(+)\&HOMA-IR(-) & TyG(-)\&HOMA-IR(+) & TyG(+)\&HOMA-IR(+) & $P$ value \\
\hline $\mathrm{N}$ & 2446 & 1530 & 304 & 305 & 307 & \\
\hline Age (year) & $59.17 \pm 8.94$ & $58.64 \pm 9.04$ & $59.58 \pm 8.62$ & $60.64 \pm 9.09$ & $59.92 \pm 8.40$ & 0.0004 \\
\hline Male, n(\%) & $968(39.6)$ & $599(39.2)$ & $148(48.7)$ & $102(33.4)$ & 119 (38.8) & 0.51 \\
\hline Current smoking, $\mathrm{n}(\%)$ & $488(20.0)$ & $311(20.3)$ & $84(27.6)$ & $40(13.1)$ & $53(17.3)$ & 0.05 \\
\hline Current drinking, $\mathrm{n}(\%)$ & $443(18.1)$ & $268(17.5)$ & $73(24.0)$ & $54(17.7)$ & $48(15.6)$ & 0.72 \\
\hline Physical activity (MET-h/wk) & $23.10(11.55-46.20)$ & $23.10(11.55-46.20)$ & $23.10(11.55-46.20)$ & $23.10(11.55-46.20)$ & $23.10(11.55-37.10)$ & 0.005 \\
\hline Highschool education, n(\%) & $1949(79.7)$ & $1255(82.0)$ & $242(79.6)$ & $222(72.8)$ & $230(74.9)$ & $<0.0001$ \\
\hline $\mathrm{SBP}(\mathrm{mmHg})$ & $135.98 \pm 20.19$ & $132.82 \pm 19.91$ & $138.04 \pm 19.29$ & $141.57 \pm 19.78$ & $143.99 \pm 19.23$ & $<0.0001$ \\
\hline $\mathrm{DBP}(\mathrm{mmHg})$ & $78.36 \pm 9.79$ & $76.84 \pm 9.70$ & $79.64 \pm 9.59$ & $80.71 \pm 9.27$ & $82.23 \pm 9.24$ & $<0.0001$ \\
\hline $\mathrm{PP}(\mathrm{mmHg})$ & $57.63 \pm 16.38$ & $55.98 \pm 16.00$ & $58.40 \pm 15.63$ & $60.86 \pm 17.07$ & $61.76 \pm 17.00$ & $<0.0001$ \\
\hline Height (cm) & $159.93 \pm 7.92$ & $159.92 \pm 7.75$ & $160.53 \pm 8.09$ & $159.30 \pm 7.81$ & $159.99 \pm 8.67$ & 0.74 \\
\hline Weight (kg) & $64.49 \pm 10.59$ & $62.17 \pm 9.62$ & $65.14 \pm 9.58$ & $69.92 \pm 11.12$ & $69.93 \pm 11.58$ & $<0.0001$ \\
\hline BMI $\left(\mathrm{kg} / \mathrm{m}^{2}\right)$ & $25.17 \pm 3.50$ & $24.28 \pm 3.19$ & $25.20 \pm 2.85$ & $27.50 \pm 3.54$ & $27.29 \pm 3.57$ & $<0.0001$ \\
\hline FPG (mmol/L) & $5.13(4.70-5.82)$ & $4.90(4.60-5.30)$ & $5.50(5.00-6.20)$ & $5.60(5.00-6.50)$ & $6.90(5.70-9.00)$ & $<0.0001$ \\
\hline Fasting insulin ( $\mu \mathrm{l} \mathrm{U} / \mathrm{ml})$ & $7.00(4.57-10.26)$ & $5.57(3.85-7.59)$ & $6.81(4.97-8.54)$ & $14.04(12.09-17.03)$ & $12.95(10.57-17.33)$ & $<0.0001$ \\
\hline Post load glucose (mmol/L) & $7.60(6.10-11.30)$ & $6.90(5.60-8.40)$ & $8.80(7.00-13.20)$ & $9.10(7.00-14.55)$ & $14.70(10.70-19.25)$ & $<0.0001$ \\
\hline $\mathrm{HbA1c}(\%)$ & $6.10(5.80-6.60)$ & $5.90(5.70-6.20)$ & $6.30(5.80-6.90)$ & $6.40(6.00-7.10)$ & $7.40(6.50-8.90)$ & $<0.0001$ \\
\hline HOMA-IR & $1.65(1.03-2.63)$ & $1.25(0.82-1.73)$ & $1.79(1.32-2.22)$ & $3.45(2.92-4.34)$ & $4.01(3.18-5.73)$ & $<0.0001$ \\
\hline Total cholesterol (mmol/L) & $5.15 \pm 0.96$ & $5.03 \pm 0.89$ & $5.42 \pm 1.03$ & $5.01 \pm 0.98$ & $5.63 \pm 1.04$ & $<0.0001$ \\
\hline Triglyceride $(\mathrm{mmol} / \mathrm{L})$ & $1.41(0.97-2.07)$ & $1.16(0.86-1.54)$ & $2.89(2.44-3.62)$ & $1.35(1.00-1.68)$ & $2.65(2.11-3.43)$ & $<0.0001$ \\
\hline HDL cholesterol (mmol/L) & $1.36 \pm 0.31$ & $1.42 \pm 0.32$ & $1.26 \pm 0.25$ & $1.29 \pm 0.29$ & $1.24 \pm 0.20$ & $<0.0001$ \\
\hline LDL cholesterol (mmol/L) & $2.39 \pm 0.68$ & $2.35 \pm 0.63$ & $2.29 \pm 0.74$ & $2.53 \pm 0.66$ & $2.52 \pm 0.80$ & $<0.0001$ \\
\hline UACR (mg/g) & $4.89(2.45-9.23)$ & $4.58(2.32-8.40)$ & $4.41(2.42-9.47)$ & $6.02(2.92-11.92)$ & $6.24(2.93-12.13)$ & $<0.0001$ \\
\hline eGFR $\left(\mathrm{ml} / \mathrm{min} / 1.73 \mathrm{~m}^{2}\right)$ & $90.90 \pm 12.75$ & $91.25 \pm 12.58$ & $90.13 \pm 12.90$ & $89.66 \pm 12.96$ & $91.22 \pm 13.20$ & 0.31 \\
\hline TyG index & $8.74 \pm 0.68$ & $8.40 \pm 0.45$ & $9.53 \pm 0.32$ & $8.68 \pm 0.41$ & $9.72 \pm 0.52$ & $<0.0001$ \\
\hline Medication use of ACEls or ARBs, $n(\%)$ & $74(3.0)$ & $33(2.2)$ & $8(2.6)$ & $13(4.3)$ & $20(6.5)$ & $<0.0001$ \\
\hline Diabetes, n(\%) & $860(35.2)$ & $310(20.5)$ & $138(46.0)$ & $167(54.8)$ & $245(80.1)$ & $<0.0001$ \\
\hline Hypertension, n(\%) & $1281(52.4)$ & $674(45.1)$ & $186(61.6)$ & $199(66.3)$ & $222(72.6)$ & $<0.0001$ \\
\hline History of MACEs, n(\%) & $213(8.7)$ & $112(7.3)$ & $37(12.2)$ & $28(9.2)$ & $36(11.7)$ & 0.007 \\
\hline MACEs at follow-up visit, n(\%) & $160(6.5)$ & $89(5.8)$ & $29(9.5)$ & $18(5.9)$ & $24(7.8)$ & 0.20 \\
\hline
\end{tabular}

Data are expressed as mean \pm standard deviation, median (interquartile range) or as $\mathrm{n}$ (\%). MET, metabolic equivalent task; BMI, body mass index; SBP, systolic blood pressure; DBP, diastolic blood pressure; PP, pulse pressure; FPG, fasting plasma glucose; HbA1c, glycated hemoglobin; LDL, low density lipoprotein; HDL, high density lipoprotein; UACR, urinary albumin-to-creatinine ratio; eGFR, estimated glomerular filtration rate; TyG, triglyceride-glucose; HOMA-IR, homeostasis model assessment of insulin resistance; ACEls, angiotensin-converting enzyme inhibitors; ARBs, angiotensin receptor blockers 
Table 2 Incidence of albuminuria using the TyG index, HOMA-IR and concordance/discordance groups

\begin{tabular}{|c|c|c|c|c|}
\hline & \multirow{3}{*}{$\begin{array}{l}\text { Cases/ } \\
\text { participants } \\
\text { (\%) }\end{array}$} & \multicolumn{3}{|l|}{ albuminuria } \\
\hline & & Model 1 & Model 2 & Model 3 \\
\hline & & OR (95\% Cl) & OR (95\% Cl) & OR (95\% Cl) \\
\hline \multicolumn{5}{|l|}{ TyG Tertiles } \\
\hline Tertile 1 & $44 / 816(5.4 \%)$ & Reference & Reference & Reference \\
\hline Tertile 2 & $58 / 815(7.1 \%)$ & $1.28(0.84-1.94)$ & $1.27(0.84-1.93)$ & $1.17(0.75-1.81)$ \\
\hline Tertile 3 & 101/815 (12.4\%) & $2.41(1.65-3.52)$ & $2.38(1.63-3.47)$ & $1.71(1.07-2.72)$ \\
\hline \multicolumn{5}{|l|}{ HOMA-IR Tertiles } \\
\hline Tertile 1 & $36 / 815$ (4.4\%) & Reference & Reference & Reference \\
\hline Tertile 2 & 66/816 (8.1\%) & $1.62(1.06-2.49)$ & $1.65(1.08-2.54)$ & $1.32(0.84-2.07)$ \\
\hline Tertile 3 & 101/815 (12.4\%) & $2.77(1.86-4.12)$ & $2.79(1.87-4.16)$ & $1.72(1.07-2.78)$ \\
\hline \multicolumn{5}{|l|}{ TyG/HOMA-IR } \\
\hline TyG(-)\&HOMA-IR(-) & $88 / 1530$ (5.8\%) & Reference & Reference & Reference \\
\hline TyG(+)\&HOMA-IR(-) & $34 / 304$ (11.2\%) & $2.09(1.37-3.21)$ & $2.06(1.34-3.16)$ & $1.69(1.04-2.75)$ \\
\hline TyG(-)\&HOMA-IR(+) & 33/305 (10.8\%) & $1.95(1.27-2.99)$ & $1.93(1.26-2.97)$ & $1.39(0.87-2.24)$ \\
\hline TyG(+)\&HOMA-IR(+) & 48/307 (15.6\%) & $3.00(2.05-4.41)$ & $2.98(2.02-4.38)$ & $1.88(1.14-3.09)$ \\
\hline
\end{tabular}

Model 1: adjusted for age and sex;

Model 2: Model $1+$ adjusted for current smoking, current drinking, education and physical activity;

Model 3: Model $2+$ adjusted for HbA1c, PP, HDL-cholesterol, LDL-cholesterol, total cholesterol, BMI and medication use of ACEls or ARBs

$\mathrm{PP}$, pulse pressure; TyG, triglyceride glucose; HOMA-IR, homeostasis model assessment for insulin resistance; OR, odds ratio; $\mathrm{Cl}$, confidence interval; $\mathrm{BMI}$, body mass index; HbA1c, glycated hemoglobin; LDL, low density lipoprotein; HDL, high density lipoprotein; ACEls, angiotensin-converting enzyme inhibitors; ARBs, angiotensin receptor blockers

tertiles, HOMA-IR tertiles and 4 concordance or discordance groups. The prevalence rates of increased newonset albuminuria were $5.4,7.1$, and $12.4 \%$ from the range of TyG index tertiles low to high, and 4.4, 8.1, and $12.4 \%$ of HOMA-IR tertiles, respectively. Compared with the low tertile, the high TyG index tertile (OR: $1.71 ; 95 \%$ CI 1.07-2.72) and the high HOMA-IR tertile (OR: 1.72, 95\% CI 1.07-2.78) had a greater risk of developing newonset albuminuria. Considering the discordance analysis, participants in TyG (+) \& HOMA-IR (-) group had a higher risk of new-onset albuminuria than those in TyG $(-)$ \& HOMA-IR (-) group after adjustment of all covariates (OR: 1.69, 95\% CI 1.04-2.75). However, there was no significant result in the risk of new-onset albuminuria in participants with low TyG but high HOMA-IR.

Similarly, as shown in Supplementary Table 1, those in TyG (+) \& HOMA-IR (-) group experienced a greater risk of incident CKD than those in TyG (-) \& HOMAIR (-) group (OR: 1.62, 95\% CI 1.01-2.58), and the participants in TyG (+) \& HOMA-IR (+) group developed the highest risk (OR: 1.95, 95\% CI 1.21-3.12) of incident CKD.

\section{Stratified analysis for associations between the concordance or discordance groups and new- onset albuminuria and CKD}

Subgroup analysis was carried out to explore the association between the concordance or discordance groups and new-onset albuminuria in enrolled participants (Fig. 2). Compared with the TyG (-) \& HOMA-IR (-) group, participants in TyG (+) \& HOMA-IR (-) group contributed to a significantly higher risk of new-onset albuminuria in patients with diabetes (OR: 1.96, 95\% CI 1.05-3.65), with hypertension (OR: 1.83, 95\% CI 1.05-3.21) and who were aged $>60$ (OR: 2.29 , 95\% CI 1.19-4.39) after multivariable adjustment. The stratified analysis was repeated for the concordance/discordance groups with the outcome of incident CKD and the results were presented in Supplementary Table 2.

\section{Discussion}

This research observed that TyG index was significantly relevant to incident albuminuria in a dose-response manner after adjusting for confounding factors in middle-aged and older participants in China. Furthermore, the discordance analysis showed that participants in TyG (+) \& HOMA-IR (-) group experienced a higher risk of incident albuminuria after full adjustment, indicating that the TyG index was more apparently relevant to incident albuminuria than the HOMA-IR. Notably, the risk of incident albuminuria was greatest among the subgroup analyses of individuals with a discordantly high TyG index, suggesting that the TyG index might be a more effective indicator in participants with metabolic abnormalities, such as diabetes, hypertension and ageing. According to what we know, this initial study 


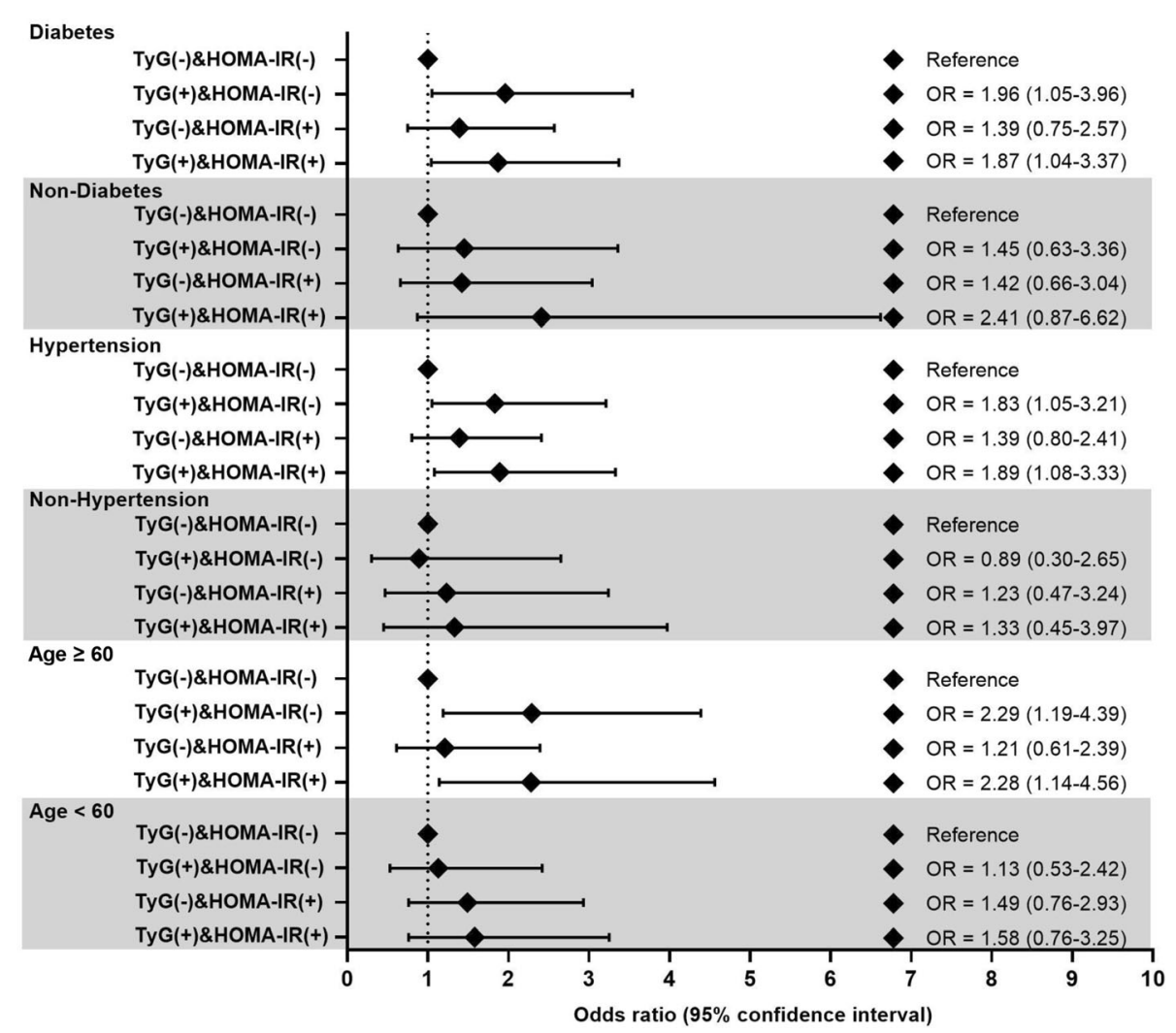

Fig. 2 Stratified analysis of the association between concordance or discordance groups of TyG index and HOMA-IR with incident albuminuria. ORs (95\% Cls) were adjusted for age, sex, current smoking, current drinking, education, physical activity, HbA1c, PP, HDL-cholesterol, LDLcholesterol, total cholesterol, BMI and medication treatment of ACEls or ARBs. TyG, triglyceride glucose; HOMA-IR, homeostasis model assessment for insulin resistance; OR, odds ratio; Cl, confidence interval; PP, pulse pressure; BMl, body mass index; HbA1c, glycated hemoglobin; LDL, low density lipoprotein; $\mathrm{HDL}$, high density lipoprotein; ACEls, angiotensin-converting enzyme inhibitors; ARBs, angiotensin receptor blockers

compared the influence of the TyG index and HOMA-IR on the risk of incident albuminuria in general population firstly.

IR in CKD individuals is closely associated with risk factors resulting in cardiovascular diseases, and the underlying mechanisms may include chronic inflammation, oxidative stress and endothelial dysfunction [21]. The results from previous studies found that patients with early-stage CKD with near-normal creatinine had defects in the insulin-mediated metabolic pathway of glucose. A retrospective cohort research carried out in Korea by Jiang et al. [22] demonstrated that IR was positively associated with the development of albuminuria in healthy individuals without diabetes. Albuminuria is an early manifestation of CKD. In addition, it is also a predictive factor of cardiovascular diseases in subjects with or without diabetes. HIEC is the "gold standard" approach for IR, where as it is not practical to use HIEC in the clinic due to its time-consuming and labourintensive nature. HOMA-IR is currently the most commonly applied clinical indicator to assess IR. A lot of researches have presented that HOMA-IR is strongly associated with the progression of albuminuria. Recently, the REACTION (Risk Evaluation of Cancers in Chinese Diabetic Individuals: A Longitudinal) study conducted in China [23] found that HOMA-IR was positively related to UACR in prediabetes or diabetes groups, but this relationship was not found in the normal glucose tolerance group. Another large prospective study showed that HOMA-IR quintiles were correlated with the incidence of CKD in adults without diabetes. In the current study, HOMA-IR was further confirmed to have a dose-response relationship with new-onset albuminuria in the general population.

Previous studies have demonstrated a close linkage between the TyG index and cardiovascular diseases. A study including healthy subjects demonstrated that an increasing TyG index was related to a greater risk of cardiovascular disease independent of diabetic status [24]. Limited researches have illustrated the relationship between TyG index and nephric disease. Only one community-based cross-sectional study [14] discovered that higher TyG index was related to elevated microalbuminuria (OR: 1.61, 95\% CI 1.22-2.13) and CKD (OR: 
1.67, 95\% CI 1.10-2.50) risk. This finding was consistent with the present study. Furthermore, data from this study supported that the TyG index might be an improved IR surrogate marker compared with HOMA-IR in the early stage of renal disease. According to the present study, participants in TyG (+) \& HOMA-IR (-) group experienced a significantly greater risk of incident albuminuria independent of traditional cardiovascular disease risk factors in discordant analysis, first elaborating the diagnostic value of the TyG index in the early stage of nephrotic damage.

Abnormal metabolic status such as diabetes and hypertension are established risk factors of cardiovascular diseases. Previous studies [25-27] have illustrated that diabetes and hypertension harmed the microvascular system, and the underlying mechanism between diabetes, hypertension and microcirculation might include hypertrophic remodelling in small vessels, endothelial dysfunction and vascular dysfunction at the capillary network. These ultimately lead to an increase in microvascular permeability to large molecules (such as albumin) and impaired insulin sensitivity. Since then, several epidemiological studies $[28,29]$ have reported the UACR as a predictive index of cardiovascular events and mortality in diabetes, hypertension and the general population. In the current study, the risk of incident albuminuria across the 4 concordance or discordance groups in different cardiovascular metabolic disorder groups was further investigated, showing that the TyG index performed more effective than HOMA-IR in identifying incident albuminuria risk in subjects with higher cardiovascular metabolic risk, such as diabetes status and hypertension status.

Ageing is related to IR to a certain content. A community-based study of participants aged $\geq 65$ years old in northern Shanghai [14] obtained a result that elevated TyG index had a greater risk of new-onset microalbuminuria or CKD. Rather than comparing the TyG index with other markers of IR in ageing participants, they revealed an linkage between the TyG index and nephric dysfunction. In this research, through the discordance analysis with HOMA-IR, the results further proved that the TyG index could recognize early renal stage in elderly individuals, which could improve the early detection of diseases.

\section{Comparisons with other studies and what does the current work add to the existing knowledge}

Previous studies have demonstrated the associations between the two indexes mentioned above with microalbuminuria or CKD separately in specific populations, such as elderly individuals or those with diabetes [14, 23]. However, this study compared the performance of TyG index with HOMA-IR in general population. Meanwhile,
TyG index implemented more effective than HOMA-IR at identifying new-onset albuminuria in subjects with metabolic disorders.

\section{Study strengths and limitations}

This current study has some strengths. This study directly compared the effectiveness of the TyG index and HOMA-IR at evaluating new-onset albuminuria risk in the general population for the first time and approved that the TyG index was better at identifying metabolic disorder subjects with new-onset albuminuria than HOMA-IR. There are also several limitations of this study that should be considered. First, this research is presented in a Chinese population aged more than 40 years old, which is not generalizable to other ethnic groups. Second, the length of the follow-up time (3.9 years follow-up) may not have been sufficient to completely capture the probable occurrence of albuminuria and CKD. Third, the widespread use of the TyG index as the best cut-off value as an alternative marker requires further research in future studies. Lastly, some underlying metabolic-related disorder factors that might influence the results were not considered in the current research, like primary triglyceride abnormalities.

\section{Conclusion}

This present study summarized that participants companying a discordantly high TyG index had a significantly greater incident albuminuria risk, especially in subjects with cardiovascular metabolic abnormalities. This conclusion supports the clinical value of the TyG index, with its readily available and reliable feature in clinical practice and could help clinicians identify the early stage of CKD in advance.

\section{Abbreviations}

ACEl: angiotensin-converting enzyme inhibitor; ADA: American Diabetes Association; ARB: angiotensin receptor blocker; BMI: body mass index; BP: blood pressure; Cl: confidence interval; CKD: chronic kidney disease; DBP: diastolic blood pressure; eGFR: estimated glomerular filtration rate; FPG: fasting plasma glucose; HbA1c: glycated haemoglobin; HDL: highdensity lipoprotein; HIEC: hyperinsulinaemic-euglycaemic clamp; HOMAIR: homeostasis model assessment of insulin resistance; IR: insulin resistance; LDL: low-density lipoprotein; PP: pulse pressure; OGTT: oral glucose tolerance test; OR: odds ratio; REACTION Study: Risk Evaluation of Cancers in Chinese Diabetic Individuals: A Longitudinal Study; SBP: systolic blood pressure;

TG: triglyceride; TyG: triglyceride-glucose; UACR: urinary albumin-to-creatinine ratio

\section{Supplementary Information}

The online version contains supplementary material available at https://doi. org/10.1186/s12944-021-01602-w.

Additional file 1. Supplementary Table 1 Incidence of CKD using the TyG index, HOMA-IR and concordance/discordance groups ( $\mathrm{N}=2448)$. Supplementary Table 2 Stratified analyses of the association between concordance/discordance groups and CKD. Supplementary Figure 1 Participant Flow Diagram of CKD outcome. Supplementary Figure 2 
Scatterplots and prevalence of discordance and concordance defined according to the upper quartile values of TyG index and HOMA-IR.

\section{Acknowledgements}

The investigators are grateful to all participants for their cooperation in the study.

\section{Authors' contributions}

Conceptualization: Jinli Gao, Mian Li, Yufang Bi; Methodology: Wei Gao, Jialu Wang, Yan Chen, Hongmei Qiao, Xiaozhong Qian, Zhuojun Xin; Formal analysis and investigation: Wei Gao, Jialu Wang, Yan Chen; Writing - original draft preparation: Wei Gao, Jialu Wang, Yan Chen; Writing - review and editing: Jinli Gao, Mian Li; Funding acquisition: Yufang Bi, Yu Xu, Min Xu, Tiange Wang; Resources: Min Xu, Zhiyun Zhao; Supervision: Jinli Gao, Mian Li, Yufang Bi. All authors read and approved the final manuscript.

\section{Funding}

This work was supported by the grants from the National Key R\&D Program of China (2017YFC1310700, 2018YFC1311800, 2018YFC1311705), the National Natural Science Foundation of China (81870560, 82070880, 81941017, 81770842, 81970706, 82022011), the Shanghai Municipal Government (18411951800), the Shanghai Shenkang Hospital Development Center (SHDC12019101, SHDC2020CR1001A, SHDC2020CR3069B), the Shanghai Jiaotong University School of Medicine (DLY201801), the Ruijin Hospital (2018CR002), and Shanghai Rising-Star Program (21QA1408100). The investigators are grateful to all participants for their cooperation in the study.

\section{Availability of data and materials}

The datasets generated during and/or analysed during the current study are not publicly available due to the data privacy policy of the cohort but are available from the corresponding author on reasonable request.

\section{Declarations}

\section{Ethics approval and consent to participate}

Due to the anonymity of the data, research ethics has been approved in previous studies, so there was no need to reapply in this study.

\section{Consent for publication}

Not applicable.

\section{Competing interests}

All authors have no conflicts of interest to declare.

\section{Author details}

'Songnan Town Community health Service Center, Baoshan District, Shanghai, China. ${ }^{2}$ Department of Endocrine and Metabolic Diseases, Shanghai Institute of Endocrine and Metabolic Diseases, Ruijin Hospital, Shanghai Jiaotong University School of Medicine, 197 Rui-Jin 2nd Road, Shanghai 200025, China. ${ }^{3}$ Shanghai National Clinical Research Center for Metabolic Diseases, Key Laboratory for Endocrine and Metabolic Diseases of the National Health Commission of the PR China, Shanghai National Center for Translational Medicine, Ruijin Hospital, Shanghai Jiaotong University School of Medicine, 197 Rui-Jin 2nd Road, Shanghai 200025, China.

Received: 15 September 2021 Accepted: 19 November 2021 Published online: 05 December 2021

\section{References}

1. Jha V, Garcia-Garcia G, Iseki K, Li Z, Naicker S, Plattner B, et al. Chronic kidney disease: global dimension and perspectives. Lancet. 2013;382(9888): 260-72. https://doi.org/10.1016/S0140-6736(13)60687-X.

2. Collaboration GBDCKD. Global, regional, and national burden of chronic kidney disease, 1990-2017: a systematic analysis for the global burden of disease study 2017. Lancet. 2020;395(10225):709-33. https://doi.org/10.1016/ S0140-6736(19)32977-0.

3. Ayodele OE, Alebiosu CO. Burden of chronic kidney disease: an international perspective. Adv Chronic Kidney Dis. 2010;17(3):215-24. https://doi.org/10.1 053/j.ackd.2010.02.001.
4. Chronic Kidney Disease Prognosis C. Matsushita K, van der Velde M, Astor BC, Woodward M, Levey AS, de Jong PE, Coresh J, Gansevoort RT: association of estimated glomerular filtration rate and albuminuria with allcause and cardiovascular mortality in general population cohorts: a collaborative meta-analysis. Lancet. 2010;375(9731):2073-81. https://doi. org/10.1016/S0140-6736(10)60674-5.

5. Mathew RO, Bangalore S, Lavelle MP, Pellikka PA, Sidhu MS, Boden WE, et al. Diagnosis and management of atherosclerotic cardiovascular disease in chronic kidney disease: a review. Kidney Int. 2017;91(4):797-807. https://doi. org/10.1016/j.kint.2016.09.049.

6. Stevens PE, Levin A. Kidney disease: improving global outcomes chronic kidney disease guideline development work group M: evaluation and management of chronic kidney disease: synopsis of the kidney disease: improving global outcomes 2012 clinical practice guideline. Ann Intern Med. 2013;158(11):825-30. https://doi.org/10.7326/0003-4819-158-11-2013 06040-00007.

7. Waheed S, Matsushita K, Sang Y, Hoogeveen R, Ballantyne C, Coresh J, et al. Combined association of albuminuria and cystatin C-based estimated GFR with mortality, coronary heart disease, and heart failure outcomes: the atherosclerosis risk in communities (ARIC) study. Am J Kidney Dis. 2012; 60(2):207-16. https://doi.org/10.1053/j.ajkd.2012.03.011.

8. Rifkin DE, Katz R, Chonchol M, Fried LF, Cao J, de Boer IH, et al. Albuminuria, impaired kidney function and cardiovascular outcomes or mortality in the elderly. Nephrol Dial Transplant. 2010;25(5):1560-7. https://doi.org/10.1093/ ndt/gfp646.

9. Fliser D, Pacini G, Engelleiter R, Kautzky-Willer A, Prager R, Franek E, et al. Insulin resistance and hyperinsulinemia are already present in patients with incipient renal disease. Kidney Int. 1998;53(5):1343-7. https://doi.org/10.104 6/j.1523-1755.1998.00898.x.

10. Kimm H, Lee SW, Lee HS, Shim KW, Cho CY, Yun JE, et al. Associations between lipid measures and metabolic syndrome, insulin resistance and adiponectin. - usefulness of lipid ratios in Korean men and women. Circ J. 2010;74(5):931-7. https://doi.org/10.1253/circj.CJ-09-0571.

11. Du T, Yuan G, Zhang M, Zhou X, Sun X, Yu X. Clinical usefulness of lipid ratios, visceral adiposity indicators, and the triglycerides and glucose index as risk markers of insulin resistance. Cardiovasc Diabetol. 2014;13(1):146. https://doi.org/10.1186/s12933-014-0146-3.

12. Vasques AC, Novaes FS, de Oliveira MS, Souza JR, Yamanaka A, Pareja JC, et al. TyG index performs better than HOMA in a Brazilian population: a hyperglycemic clamp validated study. Diabetes Res Clin Pract. 2011;93(3): e98-e100. https://doi.org/10.1016/j.diabres.2011.05.030.

13. Guerrero-Romero F, Simental-Mendia LE, Gonzalez-Ortiz M, MartinezAbundis E, Ramos-Zavala MG, Hernandez-Gonzalez SO, et al. The product of triglycerides and glucose, a simple measure of insulin sensitivity. Comparison with the euglycemic-hyperinsulinemic clamp. J Clin Endocrinol Metab. 2010;95(7):3347-51. https://doi.org/10.1210/jc.2010-0288.

14. Zhao S, Yu S, Chi C, Fan X, Tang J, Ji H, et al. Association between macro- and microvascular damage and the triglyceride glucose index in communitydwelling elderly individuals: the northern Shanghai study. Cardiovasc Diabetol. 2019;18(1):95. https://doi.org/10.1186/s12933-019-0898-X.

15. Ning $G, B i$, Wang $T, X u M, X u Y$, Huang $Y$, et al. Relationship of urinary bisphenol a concentration to risk for prevalent type 2 diabetes in Chinese adults: a cross-sectional analysis. Ann Intern Med. 2011;155(6):368-74. https://doi.org/10.7326/0003-4819-155-6-201109200-00005.

16. Lin L, Peng K, Du R, Huang X, Lu J, Xu Y, et al. Metabolically healthy obesity and incident chronic kidney disease: the role of systemic inflammation in a prospective study. Obesity (Silver Spring). 2017;25(3):634-41. https://doi. org/10.1002/oby.21768.

17. Lee PH, Macfarlane DJ, Lam TH, Stewart SM. Validity of the International Physical Activity Questionnaire Short Form (IPAQ-SF): a systematic review. Int J Behav Nutr Phys Act. 2011;8(1):115. https://doi.org/10.1186/1479-58688-115.

18. Wang $T$, Li M, Chen $B, X u M, X u Y$, Huang $Y$, et al. Urinary bisphenol a (BPA) concentration associates with obesity and insulin resistance. J Clin Endocrinol Metab. 2012;97(2):E223-7.

19. Levey AS, Stevens LA, Schmid CH, Zhang YL, Castro AF 3rd, Feldman HI, et al. A new equation to estimate glomerular filtration rate. Ann Intern Med. 2009; 150(9):604-12. https://doi.org/10.7326/0003-4819-150-9-200905050-00006.

20. Levy JC, Matthews DR, Hermans MP. Correct homeostasis model assessment (HOMA) evaluation uses the computer program. Diabetes Care. 1998;21(12): 2191-2. https://doi.org/10.2337/diacare.21.12.2191. 
21. Spoto B, Pisano A, Zoccali C. Insulin resistance in chronic kidney disease: a systematic review. Am J Physiol Renal Physiol. 2016;311(6):F1087-108. https://doi.org/10.1152/ajprenal.00340.2016.

22. Jang $\mathrm{CM}$, Hyun $\mathrm{YY}$, Lee $\mathrm{KB}$, Kim H. Insulin resistance is associated with the development of albuminuria in Korean subjects without diabetes. Endocrine. 2015;48(1):203-10. https://doi.org/10.1007/s12020-014-0242-x.

23. Gu S, Wang A, Ning G, Zhang L, Mu Y. Insulin resistance is associated with urinary albumin-creatinine ratio in normal weight individuals with hypertension and diabetes: the REACTION study. J Diabetes. 2020;12(5):40616. https://doi.org/10.1111/1753-0407.13010.

24. Li S, Guo B, Chen H, Shi Z, Li Y, Tian Q, et al. The role of the triglyceride (triacylglycerol) glucose index in the development of cardiovascular events: a retrospective cohort analysis. Sci Rep. 2019;9(1):7320. https://doi.org/10.103 8/541598-019-43776-5.

25. Rizzoni D, Porteri E, Guelfi D, Muiesan ML, Valentini U, Cimino A, et al. Structural alterations in subcutaneous small arteries of normotensive and hypertensive patients with non-insulin-dependent diabetes mellitus. Circulation. 2001;103(9):1238-44. https://doi.org/10.1161/01.CIR.103.9.1238.

26. Akerstrom $T$, Laub L, Vedel K, Brand CL, Pedersen BK, Lindqvist AK, et al. Increased skeletal muscle capillarization enhances insulin sensitivity. Am J Physiol Endocrinol Metab. 2014;307(12):E1105-16. https://doi.org/10.1152/a jpendo.00020.2014.

27. Strain WD, Paldanius PM. Diabetes, cardiovascular disease and the microcirculation. Cardiovasc Diabetol. 2018;17(1):57. https://doi.org/10.1186/ s12933-018-0703-2.

28. Sung KC, Ryu S, Lee JY, Lee SH, Cheong E, Hyun YY, et al. Urine Albumin/ Creatinine Ratio Below $30 \mathrm{mg} / \mathrm{g}$ is a Predictor of Incident Hypertension and Cardiovascular Mortality. J Am Heart Assoc. 2016;5(9):5(9). https://doi.org/1 $0.1161 / J A H A .116 .003245$

29. Inoue K, Streja E, Tsujimoto T, Kobayashi H. Urinary albumin-to-creatinine ratio within normal range and all-cause or cardiovascular mortality among U.S. adults enrolled in the NHANES during 1999-2015. Ann Epidemiol. 2021; 55:15-23. https://doi.org/10.1016/j.annepidem.2020.12.004.

\section{Publisher's Note}

Springer Nature remains neutral with regard to jurisdictional claims in published maps and institutional affiliations.

Ready to submit your research? Choose BMC and benefit from:

- fast, convenient online submission

- thorough peer review by experienced researchers in your field

- rapid publication on acceptance

- support for research data, including large and complex data types

- gold Open Access which fosters wider collaboration and increased citations

- maximum visibility for your research: over $100 \mathrm{M}$ website views per year

At $\mathrm{BMC}$, research is always in progress.

Learn more biomedcentral.com/submissions 